\title{
A Regulatory Framework for Cryptocurrency
}

\author{
JOSEPH LEE* \& FLORIAN L'HEUREUX ${ }^{* *}$
}

\begin{abstract}
The paper examines cryptocurrency in the crypto-finance ecosystem and identifies areas in which regulatory intervention is required. Part I focuses on the system and processes of cryptocurrency and identifies the features of this 'decentralised' system and the legal and regulatory implications. Part II discusses the perceived benefits and the rise of cryptocurrency to determine whether those benefits fit with regulatory objectives and contribute to the rise of cryptocurrency. Part III discusses the fall of cryptocurrency and some associated factors, particularly a lack of governance. Part IV discusses the need for regulation and governance.
\end{abstract}

\section{Keywords}

Cryptocurrency, blockchain, DLT, tokenization, cryptoasset, exchanges, investor protection

\section{Introduction}

The crypto-finance phenomenon has prompted much regulatory attention. This new, distributed system directly challenges the supervisory powers of the central banks and the regulatory structure that is currently in place. One type of crypto-finance is cryptocurrency - a facility for token payment and non-cash payment. ${ }^{1}$ Cryptocurrency is a payment product in which a person's identity does not need to be disclosed for its use as a payment method. ${ }^{2}$ It is created electronically by 'miners' who use special-

* Senior lecturer, Law School, University of Exeter, j.lee@exeter.ac.uk.

** PhD candidate, Law School, University of Exeter, fl300@exeter.ac.uk. The authors are grateful to Professor David Donald of Chinese University of Hong Kong, Professor Iris Chiu of University College London, Professor Felix Chang of University of Cincinnati, Professor Andrea Lista, Professor Joasia Luzak, and Dr Chunbo Luo of University of Exeter, Mr Matthias Bauer-Langgartner of UK FCA, Dr Nai-kuan Huang, Dr Long Chao and Dr Frank Lin of Taiwan Stock Exchange, Dr Jochen Dürr, SIX, and Sir William Blair, Queen Mary University of London for their invaluable comments. All errors remain our own.

${ }^{1}$ Philipp Hacker, Chris Thomale, Crypto-Securities Regulation: ICOs, Token Sales and Cryptocurrencies under EU Financial Law, 15 European Company and Financial Law Review 645-696 (2018). Available at SSRN: $<$ https://ssrn.com/abstract=3075820 $>$ or $<$ http://dx.doi.org/10.2139/ssrn.3075820 .

${ }^{2}$ Financial Conduct Authority, Guidance on Cryptoassets, $<$ https://www.fca.org.uk/publications/con sultation-papers/cp19-3-guidance-cryptoassets> (accessed 15 March 2019). 
ist mining software to solve complex mathematical equations, which then issue a set number of 'coins' in exchange. Cryptocurrency has been used as an alternative form of payment that does not follow traditional credit and debit card/cash systems. It has the potential to revolutionise industry and commerce. However, because cryptocurrency is an encrypted decentralised payment system, law enforcement agencies and governments do not have direct access to the data and information required for regulation. The aim of this paper is to examine cryptocurrency in the crypto-finance ecosystem and to identify areas in which regulatory intervention is required. The paper has four parts. Part I focuses on the system and processes of cryptocurrency and identifies the features of this 'decentralised' system and the legal and regulatory implications. Part II discusses the perceived benefits of cryptocurrency to determine whether they fit with regulatory objectives and contribute to the rise of cryptocurrency. Part III discusses the fall of cryptocurrency and some associated factors, particularly lack of governance. Part IV discusses the need for regulation and governance.

\section{Systems and Processes of Cryptocurrency}

\subsection{Distributed Ledger Technology (DLT) Causing Decentralisation and Disintermediation}

Cryptocurrency is a type of cryptoasset and a payment method embedded on blockchain. ${ }^{3}$ Blockchain is an algorithmic technology and a type of Distributed Ledger Technology (DLT), best known for being the underlying technology of cryptoassets. ${ }^{4}$ Cryptocurrency based on blockchain claims to be able to remove the need for a trusted third party, such as a bank or government. ${ }^{5}$ It is a database of information that is spread throughout a network (decentralisation) and protected against falsification or modification (immutability). ${ }^{6}$ Transactions that occur on the network are recorded and are tamper-proofed or tamper-recorded. To be a strong DLT, the ledger must be able to handle transactions quickly, give participants access to the ledger, timestamp transactions in the order they occurred, and rely on a consensus method to avoid compromising data. ${ }^{7}$ The aim of blockchain technology is to enable a system where participants can create, transact and record their financial activities.

3 O Cann, These are the Top 10 Emerging Technologies of 2016, <https://www.weforum.org/ agenda/2016/06/top-10-emerging-technologies-2016/> (accessed 15 March 2019); K Panetta, Gartner's Top 10 Strategic Technology Trends for 2017, <https:/www.gartner.com/smarterwithgartner/gartnerstop-10-technology-trends-2017/> (accessed 15 March 2019).

${ }^{4}$ UK Government Office for Science, Distributed Ledger Technology: Beyond Blockchain (2016).

${ }_{5}$ Merlinda Andoni et al., Blockchain Technology IN THE Energy Sector: A Systematic Review of Challenges and Opportunities 100 Renewable and Sustainable Energy Reviews 145 (2019).

${ }^{6}$ Advait Deshpande, Katherine Stewart, Louise Lepetit \& Salil Gunashekar, Distributed Ledger Technologies/Blockchain: Challenges, Opportunities and the Prospects for Standards, British Standards Institution 1 (2017).

${ }^{7}$ Gary Lilienthal, Nehaluddin Ahmad, Bitcoin: Is It Really Coinage? 24(3) Computer and Telecommunications Law Review 49-56 (2018). 


\subsection{Nodes and Consensus Rules}

Blockchain is a shared database. The transactional history is contained in blocks that are "chained" together, and transactions are verified and validated by users in the network through a process known as 'mining'. The actors in the network - the nodes - execute a common protocol (software), which is a set of rules or procedures for transmitting data between electronic devices. ${ }^{8}$ It is therefore necessary for the software that executes the protocol to operate according to identical and synchronised rules across the network. ${ }^{9}$ For Bitcoin, a type of cryptocurrency, the transaction protocol, or consensus rule, requires transactions to be in the correct data format, and with the correct signatures for the Bitcoins to be spent. As a result, a transaction output cannot be double-spent within a single blockchain. ${ }^{10}$ If one of the network nodes fails to respect the consensus rules and rejects an existing block with a specific transaction, it is denied access the system's user base and transactional traffic. ${ }^{11}$ The consensus rules were devised to ensure the robustness of Bitcoin and to avoid the network being attacked by malicious nodes. ${ }^{12}$ However, the number of coins created by the protocols is not revealed, nor is the way the coins can be claimed through the 'mining' process, or what happened after all the coins have been claimed through this 'mining' process. ${ }^{13}$ Because the mining nodes are not identified, and the way the consensus rules operate to ensure stability is not made clear, the undefined consensus rules have led to socalled 'forks' that pose a major risk to financial stability.

\subsection{Soft and Hard Forks as a Major Risk to Financial Stability}

Soft and hard ${ }^{14}$ forks in the cryptocurrency represent a major risk to financial stability and consumer protection. A soft fork is a modification of the blockchain protocol that is compatible with the older version of the blockchain. ${ }^{15}$ In this case, the old validated blocks remain compatible with the new and stricter rules. Miners and nodes

\footnotetext{
${ }^{8}$ Sara Sánchez Fernández, ICOs and Investor Protection: A Cross-Border Perspective 34(3) Journal of International Banking Law and Regulation 95-96 (2019).

9 Gianluca Salviotti, Leonardo Maria De Rossi, Nico Abbatemarco, A Structured Framework to Assess the Business Application Landscape of Blockchain Technologies, 3469 (2018).

10 Andreas M Antonopoulos, Mining and Consensus in Mastering Bitcoin: Unlocking Digital Crypto-Currencies (O’Reilly, 2014).

11 Olivier Hari, Ulysse Pasquier, Blockchain and Distributed Ledger Technology (DLT): Academic Overview of the Technical and Legal Framework and Challenges For Lawyers 5 International Business Law Journal 423, 447 (2018).

12 Rui Zhang, Rui Xue, Ling Liu, Security and Privacy on Blockchain 1 ACM Comput. Surv. (2019).

${ }_{13}$ Robby Houben, Alexander Snyers, Study Requested by the TAX3 Committee: Cryptocurrencies and Blockchain - Legal Context and Implications for Financial Crime, Money Laundering and Tax Evasion (2018). Available at: <https://www.europarl.europa.eu/cmsdata/150761/TAX3\%20Study\%20 on $\% 20$ cryptocurrencies $\% 20$ and $\% 20$ blockchain.pdf $>$

14 Jeffery Atik, George Gerro, Hard Forks on the Bitcoin Blockchain: Reversible Exit, Continuing Voice 1 Stanford Journal of Blockchain Law \& Policy 29 (2018).

${ }_{15}$ Henri Arslanian, Fabrice Fischer, The Future of Finance: The Impact of FinTech, AI, and Crypto on Financial Services (Springer, 2019).
} 
do not need to join the upgraded version of the blockchain to get access to the system's user base and transactional traffic. ${ }^{16} \mathrm{~A}$ hard fork is a major modification of the protocol that can revise any aspect of the code and whose new rules are not compatible with previous ones. ${ }^{17}$ Given the protocol's distributed nature, such modification must be incorporated into the software code held individually by each node. To ensure the effectiveness of the hard fork, a majority of nodes must adopt it. If some of the miners and nodes refuse the update, then two blockchains will exist in parallel, resulting in two different cryptocurrencies. ${ }^{18}$ The newly-created branch keeps the same blocks as the main blockchain prior to the hard fork, but will then create its own separate block history. ${ }^{19}$ Thus, each person who had owned cryptocurrency before the hard fork, will have the same amount in the new updated cryptocurrency. ${ }^{20}$ Well-known hard forks include Bitcoin Cash (BCH), from Bitcoin, or Ethereum Classic (ETC), from Ethereum. ${ }^{21}$ Consumers who purchase the coins may not appreciate the risks of forks, nor know how to update the software protocols in order to safeguard their coins.

\subsection{Three Main Categories of Blockchain and Regulatory and Legal Implications}

\subsubsection{Public Blockchains}

A public blockchain is an exchange network that is controlled by all the nodes (the participants) on a peer-to-peer basis. ${ }^{22}$ There is no barrier to participating in the network or to making transactions. Joining the network simply involves downloading software that respects the blockchain protocols. ${ }^{23}$ This software is called a 'network

\footnotetext{
${ }^{16}$ Bruno Biais, Christophe Bisière, Matthieu Bouvard, Catherine Casamatta, The Blockchain Folk Theorem (TSE Working Papers, 2018).

17 A Zamyatin, N Stifter, A Judmayer, P Schindler, E Weippl \& WJ Knottenbelt, A Wild Velvet Fork Appears! Inclusive Blockchain Protocol Changes in Practice (London: Imperial College, Austria: SBA Research, 2018).

18 Jean Bacon, Johan David Michels, Christopher Millard \& Jatinder Singh, Blockchain Demystified: A Technical and Legal Introduction to Distributed and Centralised Ledgers 25(1) Rich. J.L. \& Tech. (2018)

${ }_{19}$ Christopher Natoli, Jiangshan Yu, Vincent Gramoli, Paulo Esteves-Verissimo, Deconstructing Blockchains: A Comprehensive Survey on Consensus, Membership and Structure, arXiv:1908.08316, $<$ https://arxiv.org/pdf/1908.08316.pdf> (accessed 30 August 2019).

${ }^{20}$ Robby Houben, Alexander Snyers, Study Requested by the TAX3 Committee: Cryptocurrencies and Blockchain - Legal Context and Implications for Financial Crime, Money Laundering and Tax Evasion (2018). Available at: <https://www.europarl.europa.eu/cmsdata/150761/TAX3\%20Study\%20 on $\% 20$ cryptocurrencies\%20and\%20blockchain.pdf>.

${ }^{21}$ Lawrence J Trautman, Bitcoin, Virtual Currencies, and the Struggle of Law and Regulation to Keep Pace 102 Marquette Law Review 496 (2018); Danhui Xu, Free Money, But Not Tax-Free: A Proposal for the Tax Treatment of Cryptocurrency Hard Forks 87 Fordham Law Review 2698 (2018); AKM Najmul Islam, Matti Mäntymäki, Marja Turunen, Why do Blockchains Split? An Actor-Network Perspective on Bitcoin Splits 148 Technological Forecasting and Social Change (2019).

${ }_{22}$ Dominique Guegan, The Digital World: II-Alternatives to the Bitcoin Blockchain ? 16 Documents de Travail du Centre d'Economie de la Sorbonne 2 (2018); Dominique Guegan, Public Blockchain versus Private Blockchain 20 Documents de Travail du Centre d'Economie de la Sorbonne 3-4 (2017).

23 Richard Caetano, Learning Bitcoin (Packt Publishing Ltd, 30 October 2015).
} 
client', and it enables nodes to synchronise automatically and to interact with others using a compatible 'network client'. ${ }^{24}$ All nodes are thus in an equivalent anonymous situation in regard to their participation in the network; this is the case for the Bitcoin and Ethereum blockchains. ${ }^{25}$ Using a public chain to distribute cryptoassets makes classification of their legal status a difficult task. In addition, the creators and issuers of the cryptoassets may be anonymous, hence ascertaining the purpose of the cryptoassets issued would be difficult. Clear classification of cryptoassets is required to determine who the regulators are, which regulations apply, and what kind of tax applies to gains made from mining or selling cryptoassets. A cryptoasset could potentially be e-money (such as Libra), currency (state-backed currency), a means of exchange, a security, a commodity, or a financial derivative. ${ }^{26}$

In the UK, exchange tokens such as Bitcoin on a public chain are not considered to be a currency or money, following the previous conclusions of both the Bank of England $^{27}$ and the G20 Finance Ministers and Central Bank Governors. ${ }^{28}$ They are neither a reliable store of value due to their high volatility, nor are they widely accepted as a means of exchange or used as units of account. In the UK, fewer than 600 merchants are said to accept exchange tokens as a payment tool. ${ }^{29}$ The transferring, buying and selling of exchange tokens such as Bitcoins or Ethers, including the commercial operation of cryptoasset exchanges for these tokens, currently fall outside the regulatory perimeter. However, the Fifth Money Laundering Directive (5AMLD) $)^{30}$ that was transposed into UK law in 2019 extends regulation against money laundering and the financing of terrorism ${ }^{31}$ to entities carrying out exchange between cryptoassets and fiat currencies. It also regulates exchange between one or more forms of cryptoassets, transfer of cryptoassets, safekeeping or administration of cryptoassets

${ }^{24}$ World Bank Group, Distributed Ledger Technology (DLT) and Blockchain, <http://documents. worldbank.org/curated/en/177911513714062215/pdf/122140-WP-PUBLIC-DistributedLedger-Tech nology-and-Blockchain-Fintech-Notes.pdf> (accessed 17 March 2019).

25 Dominique Guegan, The Digital World: II - Alternatives to the Bitcoin Blockchain ?,16 Documents de Travail du Centre d'Economie de la Sorbonne 2 (2018); Dominique Guegan, Public Blockchain versus Private Blockchain 20 Documents de Travail du Centre d'Economie de la Sorbonne 3-4 (2017).

26 A Blandin, AS Cloots, H Hussain, M Rauchs, R Saleuddin, JG Allen, et al., Global Cryptoasset Regulatory Landscape Study (Cambridge: Cambridge Centre for Alternative Finance, 2019); FS Board Basel: Financial Stability Board, Crypto-asset Markets Potential Channels for Future Financial Stability Implications (2018); FCA, PS 19/22: Guidance on Cryptoassets Feedback and Final Guidance to CP $19 / 3$ (2019).

27 Bank of England, The Future of Money - speech by Mark Carney, <https://www.bankofengland. co.uk/speech/2018/mark-carney-speech-to-the-inaugural-scottish-economics-conference $>$ (accessed 17 March 2019).

${ }^{28}$ G20, Communiqué - Finance Ministers and Central Bank Governors, <https://www.mof.go.jp/ english/international_policy/convention/g20/20180722.htm (accessed 17 March 2019).

29 FCA, CP 19/3: Guidance on Cryptoassets 21 (2019).

${ }^{30}$ Directive (EU) 2018/843 of the European Parliament and of the Council of 30 May 2018 amending Directive (EU) 2015/849 on the prevention of the use of the financial system for the purposes of money laundering or terrorist financing, and amending Directives 2009/138/EC and 2013/36/EU.

31 FCA, CP 19/3: Guidance on Cryptoassets (2019). 
or instruments enabling control over cryptoassets, or participation in and provision of financial services related to an issuer's offer and/or sale of a cryptoasset. ${ }^{32}$

In the Final Guidance published by the FCA in July 2019,33 it has been decided that the division between regulated and potentially unregulated tokens should be made clearer. Unregulated tokens are now referred to as fully or partly transferable tokens, decentralised or issued centrally, that give access to current or prospective goods or services in one or multiple networks and ecosystems, or which are used as a means of exchange. ${ }^{34}$ Activities related to security tokens would fall within the regulatory perimeter, and those related to utility tokens would be subject to EMRs if they are centralised, pegged to a currency or the digital representation of fiat money stored on an electronic chip. ${ }^{35}$ Furthermore, legally established issuers of such e-money have an obligation to redeem at any moment and at par value the monetary value of the e-money. ${ }^{36}$ Similarly, in France, cryptoassets are neither regarded as a currency nor considered a means of payment by the Banque de France. ${ }^{37}$ Tokens are described in the PACTE law as immaterial items, representing one or more rights in digital form that could be issued, compiled, kept and transferred through a digital shared instrument, that allows indirect identification of the owner of the tokens. ${ }^{38}$ Bitcoin is defined as a 'unit of account', which renders both private and commercial cryptocurrency traders subject to taxation. ${ }^{39}$ The majority of countries across the EU do not recognise cryptoassets as a form of currency, except for the purposes of anti-money laundering and taxation. ${ }^{40}$

\subsubsection{Private Blockchains}

A private blockchain, in contrast, is a private network on which the 'manager' can modify the protocols and procedures for transmitting data between electronic devices. ${ }^{41}$ Participation in the network requires the network owner's permission. The participants have certain rights, and decisions made on the blockchain are made by a majority vote. ${ }^{42}$ The organisation and operating conditions of a private chain are addressed in Terms and Conditions where the parties define the roles of the technol-

Ibid.

33 FCA, PS 19/22: Guidance on Cryptoassets Feedback and Final Guidance to CP 19/3 (2019).

34 Ibid.

35 Ibid.

36 Ibid.

37 Banque de France, Les dangers liés au développement des monnaies virtuelles : l'exemple du Bitcoin (The Dangers of the Development of Virtual Currencies: The Bitcoin Example), <https:// publications.banque-france.fr/sites/default/files/medias/documents/focus-10_2013-12-05_fr.pdf > (accessed 18 March 2019).

38 PACTE Law no. 2019-486 (22 May 2019), art. 26.

39 Ibid.

40 A Blandin et al., Global Cryptoasset Regulatory Landscape Study (Cambridge: Cambridge Centre for Alternative Finance, 2019).

${ }^{41}$ Karl Wüst, Arthur Gervais, Do You Need a Blockchain? (Zurich: ETH, London: Imperial College, 2018).

42 Ibid. 
ogy provider and users. The Swift system is an example of such a chain. It is becoming common in private blockchains for the operator in charge to be officially appointed in charge of the technical platforms that support blockchain. ${ }^{43}$

\subsection{Systems and Processes of Bitcoin and Its Non-Transparency}

Bitcoin is based on a public blockchain that is usable by anybody and is not owned or controlled by an entity. ${ }^{44}$ All transactions are verified and added to the public ledger through the 'mining' process, which is also the process through which new Bitcoin units are emitted. ${ }^{45}$ Bitcoins are created in the protocol, but mining nodes can claim their ownership by solving maths questions. ${ }^{46}$ Ownership of bitcoin is established through digital keys, bitcoin addresses, and digital signatures. ${ }^{47}$ The digital keys are created and stored by users in a database called a wallet. These keys are completely independent of the bitcoin protocol and can be generated and managed by the user's wallet software on its own, even offline. ${ }^{48}$ Mining nodes are responsible for solving complicated mathematical puzzles that are part of the Bitcoin programme, the socalled 'proof of work' ${ }^{49}$ However, there are rules governing the operations of mining nodes and the designer of the mathematical puzzles is not public knowledge. As Bitcoin mining is essentially guesswork and is highly competitive, being the first miner to find the correct answer in a profitable way requires the most up-to-date hardware and/or membership in a group of miners who can combine their computing power. ${ }^{50}$ Mining nodes, acting as such central authorities, record and verify transactions in a permissionless public ledger. ${ }^{51}$ This can create an unfair situation where those who possess high computing power are able to claim ownership of newly revealed coins and, potentially control the whole network. The task of calculating the hashes and mining blocks is made difficult in order to ensure that the rate at which new blocks are created remains constant, as well as to prevent the devaluation of the cryptocur-

43 Vitalik Buterin, On Public and Private Blockchains, <https://blog.ethereum.org/2015/08/07/ on-public-and-private-blockchains/> (accessed 18 March 2019). See also Jean Bacon, Johan David Michels, Christopher Millard \& Jatinder Singh, Blockchain Demystified: A Technical and Legal Introduction to Distributed and Centralised Ledgers 25(1) Rich. J.L. \& Tech. (2018).

${ }^{44}$ Marcella Atzori, Blockchain Technology and Decentralized Governance: Is the State Still Necessary?, Journal of Governance and Regulation 7 (2017).

45 Dominique Guegan, Public Blockchain versus Private Blockchain 20 Documents de Travail du Centre d'Economie de la Sorbonne 3 (2017).

46 Ibid.

47 Andreas M Antonopoulos, Keys, Addresses in Mastering Bitcoin: Unlocking Digital CryptoCurrencies (O’Reilly, 2014).

48 Ibid.

49 Michael Crosby et al., Blockchain Technology Beyond Bitcoin, Sutardja Center for Entrepreneurship \& Technology Technical Report 10 (2015).

${ }^{50}$ Merlinda Andoni et al., Blockchain Technology in the Energy Sector: A Systematic Review of Challenges and Opportunities 100 Renewable and Sustainable Energy Reviews 146 (2019).

${ }^{51}$ See National Institute of Standards and Technology, Draft NISTIR 8202 Blockchain Technology Overview (2018). 
rency. ${ }^{52}$ Another way to achieve this aim is to fix a maximum supply of units that can be generated through mining into the blockchain algorithm. ${ }^{53}$ When all coins have been claimed, will the mining function stop or continue? It is argued that mining fees will need to be increased in order to incentivize miners to continue their crucial work of maintaining the network and verifying transactions. While the increased fee structure makes sense if Bitcoin continues its success as a speculative commodity and a store of value, the issue of fees should be approached very carefully if Bitcoin is to replace or compete with fiat currency.

\section{The Perceived Benefits of Using Cryptocurrencies and the Rise of Cryptocurrencies}

\subsection{Asset Security and Privacy Protection}

\subsubsection{Security}

It has been claimed that cryptoassets stored in an offline wallet are as difficult for other people to access as a wallet of cash, the only weak point being the user's own capacity to remember their private key. ${ }^{54}$ They cannot be counterfeited or reversed arbitrarily by the sender, as is the case with credit card charge-backs. ${ }^{55}$ Cryptocurrency uses a distributed public ledger to record transactions so that there is no private database owned and controlled by a third party; instead, there is a public transaction database in which the personal identity of each cryptocurrency owner is hidden. As a result, fraud is almost impossible if the cryptocurrency is set up properly because various nodes tracking the ledger must validate the transaction. ${ }^{56}$ This is a fundamental difference from payment networks such as Alipay, WeChat or Apple Pay, which are still tied to the banking system.

\subsubsection{Privacy}

In a credit card operation, the merchant initiates the payment and obtains access to a customer's full credit line, even if the transaction is a small one. ${ }^{57}$ This is the so-called "pull" mechanism, which means funds are pulled from a third party, such as a bank or a credit card company. ${ }^{58}$ To use a credit card online, one only needs the card number, the cardholder's name, the CCV number, and the expiration date. This personal information is repeatedly shared with online retailers who are vulnerable to hacks. In

${ }^{52}$ Ghassan O Karame, Elli Androulaki, Bitcoin and Blockchain Security (Artech House, 2016).

53 Ibid.

${ }^{54}$ House of Commons, Crypto-assets, Twenty-Second Report of Session 2017-19 (2018).

55 Arti Sharma, Ayush Anand, Digital Currency and its Risk 83 International Journal of Creative Research Thoughts 554 (2018).

56 Jennifer J Xu, Are Blockchains Immune to All Malicious Attacks? 2 Financial Innovation 25 (2016), <https://doi.org/10.1186/s40854-016-0046-5 (accessed 19 March 2019).

57 Deloitte, Real-time Payments Are Changing the Reality of Payments (2015).

58 Ibid. 
the cryptocurrency world, on the other hand, the cryptocurrency holder sends the amount to the recipient or merchant without giving any further identity information. ${ }^{59}$ Bitcoin payments are similar to wire transfers or cash transactions, where payment is "pushed" directly from one party to another, without going through another financial institution, hence the name "push" mechanism. ${ }^{60}$ However, with Bitcoin, one can have the same level of privacy as a cash transaction while being able to trace it on the blockchain.

\subsubsection{Cutting Out the Middleman}

The cryptocurrency blockchain simplifies the settlement process in real property purchases. ${ }^{61}$ As the cryptocurrency blockchain is a large property rights database, digital currency can be used to execute and enforce two-party contracts on real property. ${ }^{62}$ In this way, transactions using cryptocurrency as a payment method can cut out the middleman and thus help eliminate expensive brokerage and legal fees while enabling the completion of a contract at a future time. ${ }^{63}$ Since cryptocurrencies are accessible to anyone with access to the Internet or to a mobile phone, approximately 1.7 billion individuals in the developing world who are currently excluded from traditional exchange systems could benefit from such a system. ${ }^{64}$ Cryptocurrencies are designed for low-cost and no-fee transactions, even if most users use a third-party service, such as Coinbase, which charges fees for creating and maintaining their own Bitcoin wallets. ${ }^{65}$ This service is similar to PayPal, an online intermediary payment service that facilitates peer-to-peer payment systems. However, one major benefit of cryptocurrencies is that their owners are free to engage a third party because they do not need to depend on a centralised entity to facilitate the transaction. ${ }^{66}$ In principle, cryptocurrency owners are not subject to third-party terms of service.

59 Susanne Chishti, Tony Craddock, Robert Courtneidge, Markos Zachariadis, The PayTech Book: The Payment Technology Handbook for Investors, Entrepreneurs, and FinTech Visionaries (John Wiley \& Sons, 2020); see also Tatjana Boshkov, Blockchain and Digital Currency in the World of Finance, IntechOpen 30 (2018).

${ }^{60}$ David Birch, Before Babylon, Beyond Bitcoin: From Money that We Understand to Money that Understands Us (Do Sustainability, 15 June 2017).

${ }^{61}$ J Michael Graglia, Christopher Mellon, Blockchain and Property in 2018: At the End of the Beginning (2018).

${ }_{62}$ Tatjana Boshkov, Blockchain and Digital Currency in the World of Finance, IntechOpen 30 (2018).

${ }^{63}$ Robby Houben, Alexander Snyers, Study Requested by the TAX3 Committee: Cryptocurrencies and Blockchain - Legal Context and Implications for Financial Crime, Money Laundering and Tax Evasion (2018). Available at: <https://www.europarl.europa.eu/cmsdata/150761/TAX3\%20Study\%20 on\%20cryptocurrencies\%20and\%20blockchain.pdf>.

${ }^{64}$ Marco Lichtfous, Vivek Yadav, Valentina Fratino, Can Blockchain Accelerate Financial Inclusion Globally? (2018).

${ }^{65}$ Rainer Böhme, Nicolas Christin, Benjamin Edelman, Tyler Moore, Bitcoin: Economics, Technology, and Governance 29(2) Journal of Economic Perspectives 225 (2015).

${ }^{66}$ Malcolm Campbell-Verduyn, Bitcoin and Beyond: Cryptocurrencies, Blockchains, and Global Governance (Routledge, Taylor \& Francis Group, London, 2018). 


\subsection{The Media Hype of Cryptocurrencies}

Cryptocurrency has been the subject of considerable attention in the media in recent years for several reasons, the main one related to Bitcoin. Before Bitcoin came to the market, there were attempts at creating online currencies with ledgers secured by encryption; examples include B-Money and Bit Gold. ${ }^{67}$ However, these attempts were never fully developed, largely because of the recurrent double-spending problem, a risk associated with any decentralised digital currency that can be spent twice. ${ }^{68}$ In 2008, the global financial crisis occurred and almost led to the collapse of the world banking system. Consequently, investors lost confidence in mainstream financial markets and began to look for ways to protect their wealth and to diversify their portfolios. ${ }^{69}$ In the same year, the first blockchain was conceptualised by an unknown person or group known as Satoshi Nakamoto, in a white paper entitled 'Bitcoin - A Peer to Peer Electronic Cash System'. ${ }^{70}$ This white paper describes in detail a decentralised and secure system that is governed by its own consensus rules without an issuing authority. ${ }^{71}$ In 2009 , the Bitcoin software was made available to the general public, and the first block of the Bitcoin blockchain was created through the 'mining' process. ${ }^{72}$ The Bitcoin protocol was revolutionary for virtual currency enthusiasts because it solved the double-spending problem. ${ }^{73}$ When, in 2010, the inaugural Bitcoin sale occurred it enabled Bitcoin units to be assigned a monetary value for the first time. A computer engineer swapped 10,000 units for two pizzas. ${ }^{74}$ Bitcoin's price began to rise as demand started to increase, while still remaining below $\$ 1$ at that time. ${ }^{75}$ The chaotic global financial context, coupled with the progressive rise in the

${ }^{67}$ Bernard Marr, A Short History of Bitcoin and Crypto Currency Everyone Should Read, <https:// www.forbes.com/sites/bernardmarr/2017/12/06/a-short-history-of-Bitcoin-and-cryptocurrency-everyoneshould-read/\#27069be03f27> (accessed 21 September 2019).

${ }^{68}$ Kevin V Tu \& Michael W Meredith, Rethinking Virtual Currency Regulation in the Bitcoin Age 90 WLR 279 (2015).

${ }^{69}$ Chairman Ben S Bernanke, Stabilizing the Financial Markets and the Economy, <https://www. federalreserve.gov/newsevents/speech/bernanke20081015a.htm> (accessed 22 September 2019).

70 Satoshi Nakamoto, Bitcoin, A Peer-to-Peer Electronic Cash System, <https://Bitcoin.org/Bitcoin. pdf $>$ (accessed 22 September 2019); Arvind Narayanan, Joseph Bonneau, Edward Felten, Andrew Miller, Steven Goldfeder, Bitcoin and Cryptocurrency Technologies, A Comprehensive Introduction (Princeton: Princeton University Press, 2016).

${ }^{71}$ Satoshi Nakamoto, Bitcoin, A Peer-to-Peer Electronic Cash System, <https://Bitcoin.org/Bitcoin. pdf $>$ (accessed 22 September 2019).

72 Bernard Marr, A Short History of Bitcoin and Crypto Currency Everyone Should Read, <https:// www.forbes.com/sites/bernardmarr/2017/12/06/a-short-history-of-Bitcoin-and-cryptocurrency-everyoneshould-read/\#27069be03f27> (accessed 21 September 2019).

73 Starry Peng, Bitcoin: Cryptography, Economics, and the Future 499 University of Pennsylvania: School of Engineering and Applied Science 2 (2013); J Brito \& A Astillo, Bitcoin: A Primer for Policymakers (2013).

${ }^{74}$ John Taskinsoy, Bitcoin Mania: An End to the US Dollar's Hegemony or Another Cryptocurrency Experiment Destined to Fail?, 9 (2018). Available at SSRN: <https://ssrn.com/abstract=3311989> or $<\mathrm{http}$ //dx.doi.org/10.2139/ssrn.3311989>.

75 Andrew Tar, Bitcoin Price, Explained, <https://cointelegraph.com/explained/Bitcoin-priceexplained $>$ (accessed 26 September 2019). 
value of Bitcoin units and the lack of regulation surrounding cryptocurrency trading began to attract speculative investors. ${ }^{76}$

Bitcoin mining was lucrative in the early days of Bitcoin, as network users could mine using only the calculation power of laptops and desktops. ${ }^{77}$ However, with the exponential growth in network size between 2010 and 2011, mining cartels began to emerge and costly hardware replaced the initial hardware. ${ }^{78}$ Until 28 November 2012 , 50 Bitcoin units were awarded per block mined. ${ }^{79}$ Subsequently, when block 210,000 was reached, the reward was halved to 25 Bitcoin units, which remained profitable for miners because the price continued to rise and the energy needed to mine remained reasonable. ${ }^{80}$ Many people joined the Bitcoin network to participate in the mining race and attempt to make a profit from it. While Bitcoin and its concept increased in popularity with the power of social media and aggressive person-to-person interaction, rival cryptocurrencies - sometimes known as 'alt-coins' - such as Namecoin and Litecoin emerged. ${ }^{81}$ These alternative cryptocurrencies generally aim at improving the original Bitcoin design by offering greater anonymity, speed or other advantages. ${ }^{82}$ The price of one Bitcoin eventually reached $\$ 1,000$ in 2013 , before plummeting to approximately $\$ 300 .^{83}$ Even though Bitcoin remained marginal, increasing numbers of websites and even businesses began to accept payments in Bitcoin. ${ }^{84}$ The vast majority of these were computer hardware sales sites, but acceptance gradually expanded. ${ }^{85}$ Executives at eBay also considered offering Bitcoin as a payment method on the platform. ${ }^{86}$ A university in Cyprus allowed the payment of registration fees in Bitcoin. ${ }^{87}$

\footnotetext{
${ }^{76}$ Shaen Corbet, Brian M Lucey, Andrew Urquhart \& Larisa Yarovaya, Cryptocurrencies as a Financial Asset: A Systematic Analysis, <https://ssrn.com/abstract=3143122 (accessed 27 September 2019).

77 Tristan Greene, A Brief History of Bitcoin Mining Hardware, <https://thenextweb.com/ hardfork/2018/02/02/a-brief-history-of-Bitcoin-mining-hardware/> (accessed 27 November 2019).

78 Katalyse.io, What is the Bitcoin Hype All About?, <https://hackernoon.com/what-is-the-Bitcoinhype-all-about-1ae5f9251367> (accessed 27 November 2019).

79 Melvin Draupnir, What is the Bitcoin Mining Block Reward?, <https://www.Bitcoinmining.com/ what-is-the-Bitcoin-block-reward/> (accessed 28 November 2019).

80 Ibid.

${ }^{81}$ RE Kadyrov, IV Prokhorov, Regulating Cryptocurrencies: New Challenges to Economic Security and Problems Created by Individuals Involved in the Schemes of Laundering CryptocurrenciesGenerated Profits, Moscow Engineering Physics Institute 2 (2018).

${ }^{82}$ Krishna Kumar Thakur, Dr. GG Banik, Cryptocurrency: Its Risks and Gains and the Way Ahead 9 IOSR Journal of Economics and Finance 39 (2018).

${ }_{83}$ Bernard Marr, A Short History of Bitcoin and Crypto Currency Everyone Should Read, <https:// www.forbes.com/sites/bernardmarr/2017/12/06/a-short-history-of-Bitcoin-and-cryptocurrency-everyoneshould-read/\#27069be03f27> (accessed 21 September 2019).

${ }^{84}$ Rainer Böhme, Nicolas Christin, Benjamin Edelman, Tyler Moore, Bitcoin: Economics, Technology, and Governance 29(2) Journal of Economic Perspectives 224 (2015).

85 Ibid.

${ }^{86}$ Rodolphe Durand, Nina Granqvist, Anna Tyllström, From Categories to Categorization: Studies in Sociology, Organizations and Strategy at the Crossroads, 193 (Emerald Group Publishing, 2017).

87 SN Jha, 2015 Current Affairs for IAS, UPSC, PCS, SSC, IBPS Exams, 136 (Kalinjar Publications, 2015).
} 
The publicity around this new technology was boosted in October 2015 by The Economist's issue entitled 'Blockchain, the Trust Machine', which argued that blockchain could transform the way the economy works. ${ }^{88}$ Psychological factors played an important role in this hype, and investors' speculation ${ }^{89}$ led to a one-sided demand with a peak of inflated expectations. Expectations inflated when cryptocurrency investors boasted about their skyrocketing returns while ignoring negative factors, such as the lack of regulation, the extreme volatility of the market or the use of cryptocurrencies for illicit and criminal activities. Mainstream media coverage of Bitcoin also played a role in exaggerating its potential..$^{90}$ Cryptocurrency had changed from a method of payment to a speculative investment and a tool for money laundering.

\section{Lack of Governance and the Fall of Cryptocurrencies: The Bitcoin Example}

Prices of cryptocurrencies eventually crashed on 17 December 2017 when Bitcoin reached its peak price of $\$ 19,511 .{ }^{11}$ While it was anticipated that the bubble might burst, the price of Bitcoin did not fall to zero. The excessive interest in Bitcoin was characterised by speculative demand. Studying the way in which interest in Bitcoin has faded prompts a focus on those elements that may have caused a progressive disinterest or even mistrust by investors. First, as the media publicity surrounding cryptocurrencies increased, they started to become a target for hacks and frauds which goes against the claimed benefits of cryptocurrency-asset security. Speculative pressure became a source of price volatility and posed a threat to blockchain development, while the use of cryptocurrencies for criminal and illicit activities seriously compromised its reputation and rebutted the claim that the blockchain is transparent. Lack of regulation was a significant factor in associating Bitcoin with uncertainty and untrustworthiness and consequently challenged the credibility of the 'consensus system' on blockchain.

\subsection{Cryptoassets, a Target for Hacks and Frauds}

\subsubsection{Risks to Value Linked to the Lack of Security of Exchanges}

Cryptoasset exchanges are attractive targets for hackers because they are less difficult to hack than blockchain protocols and are less distributed than wallets. ${ }^{92}$ Any weak-

\footnotetext{
88 The Economist, Blockchain, The Trust Machine (2015).

89 Ibid.

${ }_{90}$ Michal Polasik, Anna Piotrowska, Radoslaw Kotkowski, Tomasz Piotr Wisniewski, Price Fluctuations and the Use of Bitcoin: An Empirical Inquiry 20(1) International Journal of Electronic Commerce 9, 49 (2015).

${ }^{11}$ Galina Hale, Arvind Krishnamurthy, Marianna Kudlyak, Patrick Shultz, How Futures Trading Changed Bitcoin Prices, <https:/www.frbsf.org/economic-research/publications/economic-letter/2018/ may/how-futures-trading-changed-Bitcoin-prices/> (accessed 7 October 2019).

92 Financial Inclusion Global Initiative - Security, Infrastructure and Trust Working Group, Security Aspects of Distributed Ledger Technologies (2019).
} 
ness in design means that cryptocurrency exchange is vulnerable and which may provide a centralised failure point. ${ }^{93}$ Since 2017 , hackers such as the infamous North Korean hacker crew dubbed "Lazarus" have exploited these flaws for huge gains amounting to hundreds of millions of dollars. ${ }^{94}$ However, even if there is no such centralised failure point in the design of cryptoassets, it does not mean that their consensus cannot be used by a malicious group of miners representing the majority of the network's mining hash rate or computing power. ${ }^{95}$ In this case, the majority group could alter transaction history and prevent the validation of new transactions. Moreover, cryptocurrency exchanges do not benefit from the same protection as stock exchanges. While stock exchanges and cryptocurrency exchanges share the key function of acting as platforms on which assets are traded, cryptocurrency exchanges differ in that they hold investors' assets in a common wallet that is usually connected to the Internet and not owned by investors. ${ }^{96}$ While the safe-keeping of assets is not a function undertaken by stock exchanges, this is not the case with cryptocurrency exchanges. Most cryptocurrency exchanges also offer safe-keeping of the private cryptographic keys of a large number of their users ${ }^{97}$ and a private key allows a user to unlock a specific address and to obtain control of the clients' digital assets. ${ }^{98}$ If such a cryptocurrency exchange is hacked, then the hacker could copy the database of private keys, access all the specific addresses and immediately siphon out all the available funds. ${ }^{99}$ If most of the funds are stored in a 'hot' wallet that is connected to the Internet, a hacker who gains access to the wallet could easily steal the funds. ${ }^{100}$ Furthermore, although stock exchanges are required to hold security insurance to cover the risk of loss, cryptocurrency exchanges are not. ${ }^{101}$ Only Coinbase and Gemini offer security insurance on cash deposits. ${ }^{102}$ Thus, when assets are in a common

93 Ibid.

94 Neil Mathew, \$571 Million: Notorious North Korean Hacker Group Has Stolen a Fortune’, $<$ https://www.ccn.com/571-million-notorious-north-korean-hacker-group-has-stolen-a-fortune-incryptocurrency> (accessed 17 March 2019).

95 Hossein Nabilou, André Prüm, Ignorance, Debt, and Cryptocurrencies: The Old and the New in the Law and Economics of Concurrent Currencies 5(1) Journal of Financial Regulation 29, 63 (2019).

${ }^{96}$ Dennis Chu, Broker-Dealers for Virtual Currency: Regulating Cryptocurrency Wallets and Exchanges 118(8) Columbia Law Review 2323, 2360 (2018).

97 Robby Houben, Alexander Snyers, Study Requested by the TAX3 Committee: Cryptocurrencies and Blockchain - Legal Context and Implications for Financial Crime, Money Laundering and Tax Evasion (2018). Available at: <https://www.europarl.europa.eu/cmsdata/150761/TAX3\%20Study\%20 on $\% 20$ cryptocurrencies $\% 20$ and $\% 20$ blockchain.pdf $>$

98 A Blandin et al., Global Cryptoasset Regulatory Landscape Study (Cambridge: Cambridge Centre for Alternative Finance, 2019).

99 Ibid.

${ }^{100}$ Paul Vigna, Michael J Casey, The Age of Cryptocurrency: How Bitcoin and Digital Money Are Challenging the Global Economic Order (St. Martin's Publishing Group, 2015).

${ }_{101}$ House of Commons, Crypto-assets, Twenty-Second Report of Session 2017-19 (2018).

102 See Gemini's User Agreement, Digital Asset Insurance, <https://gemini.com/legal/useragreement\#digital-asset-insurance $>$ (accessed 3 December 2019); Coinbase, How is Coinbase Insured?, $<$ https://support.coinbase.com/customer/portal/articles/1662379-how-is-coinbase-insured-> (accessed 3 December 2019). 
wallet, there is a high risk of theft. ${ }^{103}$ The best way for investors to protect their assets is to keep their digital tokens away from exchanges and to store them offline in 'cold' storage. ${ }^{104}$ This move is impractical for frequent traders who hold cryptocurrency across several exchanges. For exchanges which choose to keep most of their assets in offline storage wallets, they must ensure that there is more than one person responsible for the wallets and their corresponding keys, or set up a back-door mechanism. To avoid the single point of failure, cryptocurrency exchanges should keep cryptoassets in a cold wallet that is offline most of the time and require permission from multiple parties for any change in the wallet. This process is similar to the way the central securities depository operates. A smart contract that allows automatic disclosure of the cold wallet's private key to a designated trustworthy person should also be coded, in order to prevent customers' funds from being permanently lost. ${ }^{105}$ Cryptocurrency exchanges should also be subject to the requirements of securities trading venues and securities depositories to ensure market safety and investor protection.

\subsubsection{Value Risks Linked to Frauds}

Cryptoassets, taken in a broader sense than cryptocurrency, have experienced very strong growth since the multiplication of exchange platforms. In 2013, the market was mainly based on 14 cryptoassets, ${ }^{106}$ but by 2019 , there were nearly 1,500 in circulation, $50 \%$ of which are simple tokens created on networks. ${ }^{107}$ However, this great expansion has been accompanied by an equivalent boom in fraud: more than $80 \%$ of ICOs (Initial Coin Offerings) conducted in 2017 were identified as frauds. ${ }^{108}$ An ICO is similar to a direct listing because the business sells tokenised securities or interests directly to the public without regulatory approval, underwriters or intermediaries. Since there is a widespread lack of understanding about blockchain technology, combined with the system's opacity, fear of missing gains, and uncontrolled social media hype, ICO fraudsters create fake crypto-coins and offer them to investors by promising unrealistic speculated targets and profit returns in their white papers. ${ }^{109}$ Such white papers usually contain inaccurate information or stolen content and do not give the details of the team behind the venture or provide a clear roadmap. ${ }^{110}$ In some instances, fraudsters do not even provide a white paper.

103 Patrick McCorry, Malte Möser, Syed Taha Ali, Why Preventing a Cryptocurrency Exchange Heist Isn't Good Enough, Security Protocols Workshop 3 (2018).

104 European Securities and Markets Authority, Advice - Initial Coin Offerings and Crypto-Assets (2019); Asia Securities Industry and Financial Markets Association, ASIFMA Best Practices for Digital Asset Exchanges (2018).

105 Timothy G Massad, It's Time to Strengthen the Regulation of Crypto-Assets (Economic Studies at Brookings, 2019).

106 Satis Group, Cryptoasset Market Coverage Initiation: Technical Underpinnings, <https:// research.bloomberg.com/pub/res/d2246jsnqusjYSeacPbQc2IjVIw> (accessed 3 October 2019).

107 Ibid.

108 Ibid.

109 Paul Barnes, Cryptocurrency and its Susceptibility to Speculative Bubbles, Manipulation, Scams and Fraud, Munich Personal RePEc Archive 15, 17 (2018).

110 David Florysiak, Alexander Schandlbauer, The Information Content of ICO White Papers, European Financial Management Journal 7, 8 (2019). 
Those who attempt to defraud investors have also been known to develop counterfeit smart contracts and target exchanges that appear to list their fake digital assets. ${ }^{111}$ These counterfeit products appear with falsely high prices through volume generation by fraudsters. Prices then crash once the assets are sold to investors. The estimated amount lost from hacks and frauds between 2011 and 2018 was approximately $\$ 2.3$ billion USD, and most of these thefts occurred on exchanges, although some relate to offline storage wallets. ${ }^{112}$ These recurrent and significant hacks and thefts have begun to arouse mistrust and have increased investors' concerns about the safety of ICOs, which has also had a negative impact on cryptocurrency growth. Despite the inherent uncertainty about ICOs and the general decline in the market, fundraising for crypto projects steadily increased in the early part of $2018 .{ }^{113}$ However, this growth only lasted until the end of the first half of 2018 before decreasing significantly in number and volume. ${ }^{114}$ This decrease can be explained by the progressive shift from ICOs to regulated STOs, and a global shift from quantity to quality. ${ }^{115}$ STOs are a more mature and regulated form of ICO as the underlying tokens provide different financial rights such as dividends and mainly focus on accredited investors by imposing higher market entry barriers to comply with regulations. ${ }^{116}$ Regulated cryptomarkets can improve user confidence and prevent fraud.

\subsection{Speculative Pressure, a Source of Price Volatility and Danger for Blockchain Development}

The main problem with cryptocurrency may still lie in price volatility and the fact that most Bitcoin owners consider it an investment currency. ${ }^{117}$ Many consumers see cryptoassets as a fast-track to easy wealth, without fully understanding what they are purchasing. ${ }^{118}$ Their behaviour is influenced by the advice of a few influential recommendations rather than mainstream media or official sources of information. ${ }^{119}$ The increasing speculative pressure that investors have placed on cryptocurrency, often irrationally, may have acted to kill the innovative nature of blockchain development. When speculation results in volatile market conditions, it is difficult to experiment

111 Christof Ferreira Torres, Mathis Steichen, Radu State, The Art of The Scam: Demystifying Honeypots in Ethereum Smart Contracts (2019). Available at: <https://arxiv.org/pdf/1902.06976.pdf>.

112 The New York Times Editorial Staff, Cryptocurrencies: Bitcoin, Blockchain and Beyond (The Rosen Publishing Group, Inc, 15 December 2018).

113 Satis Group, Cryptoasset Market Coverage Initiation: Technical Underpinnings, <https:// research.bloomberg.com/pub/res/d2246jsnqusjYSeacPbQc2IjVIw> (accessed 3 October 2019).

114 Strategy\& PwC, Crypto Valley, 4th ICO/STO Report - A Strategic Perspective (2019).

115 Ibid.

116 Michael Mendelson, From Initial Coin Offerings to Security Tokens: A U.S. Federal Securities Law Analysis 22(1) Stanford Technology Law Review 52 (2019).

117 Giancarlo Giudici, Alistair Milne, Dmitri Vinogradov, Cryptocurrencies: Market Analysis and Perspectives, Journal of Industrial and Business Economics 8 (2019).

118 FCA, Cryptoassets: Ownership and Attitudes in the UK-Consumer Survey Research Report (2019).

119 Ibid. 
with the technology to promote a more successful and secure use of cryptocurrency. The technology struggles to keep pace with developments.

It was argued that the fall of Bitcoin was directly linked to the launch of a futures market on the Chicago Mercantile Exchange. ${ }^{120}$ Because there was no market for Bitcoin derivatives, it was extremely difficult to bet on the decline in Bitcoin prices, as such bets usually take the form of short selling. ${ }^{121}$ Thus, futures contracts gave institutional investors the ability to manipulate the crypto market and suppress the over-exaggeration of Bitcoin value to better align the asset with the business model of mainstream financial services. The FCA plans to make derivative contracts of cryptocurrency illegal and unenforceable in order to protect retail investors from high volatility and risks. ${ }^{122}$ By making these contracts legal, regulators would have given legitimacy to cryptocurrency, and allowed it to taint the safety of derivatives market. Furthermore, the gambling sentiment of the financial market should not be encouraged, as most consumers cannot reliably value derivatives based on unregulated cryptoassets. ${ }^{123}$ Finally, a derivative contract using cryptocurrency as a benchmark cannot currently be enforced. While it is essential to warn and support retail customers, a ban of derivatives contracts would currently be preferable for the reasons detailed above. Moreover, the market abuse regime should apply to cryptocurrency markets in order to enhance market integrity and investor protection.

\subsection{Criminal and Illicit Activities, a Reputation Killer for Cryptocurrencies}

Because of the initial lack of regulation of the crypto space, many commentators have portrayed Bitcoin as a medium for criminal and illicit activities. ${ }^{124}$ This response is largely due to the Silk Road website, which is an online market place on the dark web for buying and selling drugs, weapons and other illicit articles that uses Bitcoin as its main currency. ${ }^{125}$ Illicit buying and selling was simple for criminals, especially in 2011, when the monetary value of a Bitcoin unit was $1 \$ .{ }^{126}$ Although this website contributed to the rising popularity of Bitcoin, at the same time, it tainted the reputa-

\footnotetext{
${ }^{120}$ Neil Gandal, Hanna Halaburda, Working Paper: Competition in the Cryptocurrency Market, 33 Bank of Canada Working Paper 15 (2014).

${ }^{121}$ Galina Hale, Arvind Krishnamurthy, Marianna Kudlyak, Patrick Shultz, How Futures Trading Changed Bitcoin Prices, <https:/www.frbsf.org/economic-research/publications/economic-letter/2018/ may/how-futures-trading-changed-Bitcoin-prices/> (accessed 7 October 2019).

122 FCA, FCA Proposes Ban on Sale of Crypto-derivatives to Retail Consumers, <https://www. fca.org.uk/news/press-releases/fca-proposes-ban-sale-crypto-derivatives-retail-consumers $>$ (accessed 19 November 2019).

123 Ibid.

124 Samantha Douma, Bitcoin: The Pros and Cons of Regulation, Universiteit Leiden 13 (2016); Joe Mont, Very Real Regulations Issued on Virtual Currencies 10 (112) Compliance Week (2013).

125 Joseph Cox, Chapter 4 - Staying in the Shadows: The Use of Bitcoin and Encryption in Cryptomarkets in European Monitoring Centre for Drugs and Drug Addiction, The Internet and Drug Markets: Insight, 21 (Publications Office of the European Union, Luxembourg, 2016).

126 Rainer Böhme, Nicolas Christin, Benjamin Edelman, Tyler Moore, Bitcoin: Economics, Technology, and Governance 29(2) Journal of Economic Perspectives 222 (2015).
} 
tion of cryptocurrency, even after the website had been shut down by the Federal Bureau of Investigation (FBI) and the criminals convicted by the US Federal Court in Manhattan. ${ }^{127}$ As cryptoassets such as Bitcoin are in essence digital and there is no physical location linked to a cryptocurrency wallet, they quickly became the favourite payment method for cyber-criminals using Ransomware. ${ }^{128}$ Various studies and official sources show that the use of cryptoassets for illicit activities is small compared to the use of fiat. The most recent instance concerned Denmark's biggest bank, whose CEO had to resign following a $\$ 235$ billion USD money laundering scandal. ${ }^{129}$ Furthermore, using Bitcoin does not make criminals' transactions untraceable because the veil of anonymity on blockchain is potentially breakable, whereas cash is often traceless. ${ }^{130}$ However, the money laundering regime needs to apply to activities related to cryptocurrencies, both on public or private chains, in order to prevent financial market from being tainted with criminal activities. Cryptocurrency exchanges ought to be required to conduct 'Know-your-customer' processes.

\subsection{Lack of Regulation, a Cause of Uncertainty and Untrustworthiness}

Discussions about blockchain are dominated by its applications in finance and business. It is perceived mostly as a threat to the current system, and it suffers from a consequent lack of trust. ${ }^{131}$ In 2018, at least $60 \%$ of Americans, Europeans and Australians were aware of cryptoassets, but only $8 \%$ and $9 \%$ of Europeans owned cryptocurrency. ${ }^{132}$ Regulatory interventions have an impact not only on the price of cryptoassets but also on blockchain success. ${ }^{133}$ The uncertain relationship between blockchain and law leads to scepticism and confusion. Some critics also see this technology as a haven for criminal activity and a way to avoid legal accountability. ${ }^{134}$ This scepticism led Chinese regulators to shut down several Bitcoin exchanges and ban ICOs in 2017, causing a Bitcoin price drop of $29 \%$ in 24 hours. ${ }^{135}$ The lack of regula-

127 Ibid.

128 Ibid.

129 Will Martin, The CEO of Denmark's Biggest Bank is out after a \$235 Billion Money-Laundering Scandal, <https://www.businessinsider.com.au/danske-bank-ceo-resigns-estonian-money-launderingscandal-2018-9\#2MyHdee8KaPPx72k.99> (accessed 11 October 2019).

130 Franscica Moreno, Shivangee Trivedi, Staying Anonymous on the Blockchain: Concerns and Techniques, $<$ https://securingtomorrow.mcafee.com/mcafee-labs/staying-anonymous-on-the-blockchainconcerns-and-techniques/> (accessed 11 October 2019).

131 Peter D De Vries, An Analysis of Cryptocurrency, Bitcoin, and the Future 1(2) International Journal of Business Management and Commerce 6 (2016).

132 ING, International Survey - Mobile Banking 2018 - Cryptocurrency, <https://www.ezonomics. com/ing_international_surveys/mobile-banking-2018-cryptocurrency/> (accessed 17 October 2019).

133 Raphael Auer, Stijn Claessens, Regulating Cryptocurrencies: Assessing Market Reactions, BIS Quarterly Review 55 (2018).

${ }^{134}$ Kevin Werbrach, Trust, But Verify: Why the Blockchain Needs the Law 33 Berkeley Technology Law Journal 489 (2018).

135 Rishi Iyengar, China's Bitcoin Crackdown Forces Exchanges to Close. The price is crashing, $<$ https://money.cnn.com/2017/09/15/technology/china-Bitcoin-exchanges-prices-crash/index.html> (accessed 14 October 2019); Alicia Cameron, Kelly Trinh, Four Factors Driving the Price of Bitcoin, 
tion has also prevented compliant ICO projects from working. ${ }^{136}$ Japan has recognised Bitcoin as a legal tender, enabling Bitcoin to be used as an official form of payment to pay a public or private debt or meet a financial obligation. ${ }^{137}$ Such legal recognition pushed Bitcoin prices up by $2 \%$ in 24 hours and increased the price globally by $160 \%$ for the next two months. ${ }^{138}$ In the US, August 2018 was marked by the decision of the US Securities \& Exchange Commission (SEC) to reject no less than nine proposals for Exchange-Traded Funds that are indexed on Bitcoin. ${ }^{139}$ The SEC announced the rejection of 9 ETF projects from three different issuers, Direxion, GraniteShares and ProShares ${ }^{140}$ for lack of assurances concerning product safety, in particular the risk of fraud and manipulation. ${ }^{141}$

Computer programmes using various indicators to recognise trends and automatically execute trades to manipulate the price of Bitcoin are not new. ${ }^{142}$ Market manipulation creates unstable market conditions and artificially inflated volumes across exchanges. ${ }^{143}$ Algorithm traders or major traders usually use three techniques to manipulate a regulated market: pump and dump, ${ }^{144}$ order spoofing ${ }^{145}$ or wash trading. ${ }^{146}$ Although this problem continues to be subject to frequent regulatory review, the SEC was called to reconsider its rules, regulations, and guidelines so as to ensure that they are still working as intended to accomplish the SEC's mission. ${ }^{147}$ Risk management strategies and the ways in which crypto trading platforms fulfil customer responsi-

$<$ http://theconversation.com/four-factors-driving-the-price-of-Bitcoin-87244> (accessed 5 October 2019).

136 Alfred Ruoxi Zhang et al., The Regulation Paradox of Initial Coin Offerings: A Case Study Approach 2(2) Policy and Practice Reviews (2019).

${ }_{137}$ Marta Gonzalez, Blockchain in Japan (EU-Japan Centre for Industrial Cooperation, 2018).

138 Ibid. at 31.

139 Ana Alexandre, US SEC to Review Rejection of Nine Bitcoin ETF Applications, <https:// cointelegraph.com/news/us-sec-to-review-rejection-of-nine-Bitcoin-etf-applications $>$ (accessed 14 October 2019).

140 See, for instance, Securities and Exchange Commission, Self-Regulatory Organizations; NYSE Arca, Inc.; Order Disapproving a Proposed Rule Change to List and Trade the Shares of the ProShares Bitcoin ETF and the ProShares Short Bitcoin ETF, <https:/www.sec.gov/rules/sro/ nysearca/2018/34-83904.pdf> (accessed 15 October 2019).

141 Ibid.

142 Matthew Hougan, Hong Kim, Micah Lerner, Economic and Non-Economic Trading in Bitcoin: Exploring the Real Spot Market For The World's First Digital Commodity (SEC, 2019).

143 Ibid. at 33-34.

144 A group of investors buy huge quantities of a cryptocurrency to artificially inflate the course, hoping to create around this speculative bubble a feeling of a "fear of missing out". When the goal is achieved, i.e., when the operation manages to attract enough new buyers to artificially raise the price, the actor who until now played the role of "pumper" then suddenly becomes a "dumper" and sells its assets before the others to obtain maximum benefits.

${ }^{145}$ An individual creates fake purchase orders with no real intention to act in order to create an illusion about the market's health.

146 A trader or group of traders buys and sells its own orders on a particular asset to increase the trading volume and create a false and exaggerated interest in this asset.

${ }^{147}$ Elad Roisman, Statement at the Senate Committee on Banking, Housing, and Urban Affairs (24 July 2018). 
bilities vary greatly. ${ }^{148}$ Potential conflicts of interest, a lack of initiatives to tackle market manipulation and abusive trading, and insufficient protection for customers' funds were also identified as major concerns. ${ }^{149}$ Finally, retail investors are the most disadvantaged by the business model of exchanges or the types of products and services developed in crypto trading. ${ }^{150}$ The business model of exchanges consists in the maker-taker fee model which encourages market liquidity by rewarding the makers, whose orders exist on the order book prior to the trade, with a fee discount. ${ }^{151}$ By contrast, the takers, whose orders match the makers' ones, pay a higher fee for removing the liquidity created by makers. ${ }^{152}$

Regulatory concerns, as well as the closing of technological and financial channels by private institutions, ${ }^{153}$ are commonly presented as causes of the cryptocurrency downturn. The market has been highly sensitive to any news coming from the financial sector or regulators since the start of 2018, whether true or false. It is submitted that cryptocurrency trading platforms should act as a gatekeeper against market manipulation to protect the users and the investors, as well as the cryptocurrency market's integrity.

\section{The Need for Regulation and Governance and a Possible Approach}

Cryptoassets suffer from a lack of trust. This trust could be brought back by regulation, but approaches taken by regulators to date are diverse and sometimes even contradictory, which is particularly obvious in the crucial issue of the categorisation of cryptoassets.

\subsection{Regulation, a Potential Solution to the Lack of Trust in Cryptoassets}

This section will first discuss the rationale for a legal and regulatory framework as a necessary path to providing confidence and then identify major terminology problems in governing a constantly evolving technology. Finally, the substantial problems associated with trying out new methods of regulation in a dispersed architecture will be examined.

148 Barbara D Underwood, Virtual Markets Integrity Initiative - Report, <https://ag.ny.gov/sites/ default/files/vmii_report.pdf?mod=article_inline> (accessed 9 December 2019).

149 Ibid.

150 Ibid.

151 Stanislav Dolgopolov, Linking the Securities Market Structure and Capital Formation: Incentives for Market Makers? 16(1) University of Pennsylvania Journal of Business Law 37 (2013).

152 Ibid. at 38.

153 Alice Woodhouse, Bitcoin Drops on Report Goldman Sachs Shelves Trading Desk Plans, $<$ https://www.ft.com/content/59a16554-b16e-11e8-8d14-6f049d06439c > (accessed 16 October 2019); Duncan Riley, Bitcoin Price Drops as Major Banks Ban Cryptocurrency Credit Card Purchases, $<$ https://siliconangle.com/2018/02/04/Bitcoin-price-drops-major-banks-ban-cryptocurrency-credit-cardpurchases/> (accessed 17 October 2019). 


\subsubsection{The Risky but Necessary Path Towards a Legal and Regulatory Framework} Regulation may seem to be an unwanted element in the development of the decentralised Bitcoin community ${ }^{154}$ but it is necessary if cryptocurrency is to grow and become more widely accepted. Smart regulation and taxation are expected to add legitimacy, to spur investment by big players and to bring Bitcoin into conformity with existing monetary systems. ${ }^{155}$ The risk of hasty regulation is that it might hinder innovation or stifle blockchain development at an early stage solely for purposes of public policy. ${ }^{156}$ Countries such as the UK, the US, Japan, and China currently recognise the value of distributed ledger technology, and their regulations are mostly focused on cryptoassets and ICOs. Their emphasis is on crypto money laundering, tax evasion, and consumer protection from financial fraud and market manipulation. ${ }^{157}$ However, it is suggested that consumer protection law should not only apply to the cases of financial fraud and market manipulation, but also to consumer contracts in which cryptoassets have been used as a means of payment. For instance, sale of goods contracts should be protected by the implied terms and remedies under the Sale of Goods Act 1979 if cryptocurrency is used to pay for the goods. ${ }^{158}$ Consumers should be warned about the associated risks before contracting, and if a merchant fails to deliver the goods, the consumer should be entitled to compensation in fiat currency. The decision to regulate applications of the technology rather than the technology itself seems preferable to foster its development. Light-handed regulation of blockchain that is neutral with regard to the different techniques used is the best way to encourage future development. ${ }^{159}$

\subsubsection{Terminology Problems in Governing a Constantly Evolving Technology}

Global regulators face difficulties in understanding and adequately governing blockchain due to the rapid evolution of the cryptocurrency space and the inconsistent

154 Samantha Douma, Bitcoin: The Pros and Cons of Regulation, Leiden University Repository 30 (2016).

155 Ibid. at 39.

156 OECD, The Policy Environment for Blockchain Innovation and Adoption: 2019 OECD Global Blockchain Policy Forum Summary Report, <https://www.oecd.org/finance/2019-OECD-GlobalBlockchain-Policy-Forum-Summary-Report.pdf $>$ (accessed 9 December 2019).

157 Robby Houben, Alexander Snyers, Study Requested by the TAX3 Committee: Cryptocurrencies and Blockchain - Legal Context and Implications for Financial Crime, Money Laundering and Tax Evasion (2018). Available at: <https://www.europarl.europa.eu/cmsdata/150761/TAX3\%20Study\%20 on\%20cryptocurrencies\%20and\%20blockchain.pdf $>$.

158 The LawTech Delivery Panel, Legal Statement on Cryptoassets and Smart Contracts (2019). Available at: <https://35z8e83m1ih83drye280o9d1-wpengine.netdna-ssl.com/wp-content/ uploads/2019/11/6.6056_JO_Cryptocurrencies_Statement_FINAL_WEB_111119-1.pdf $><$ https:// technation.io/about-us/lawtech-panel/> (accessed 20 January 2020); Miklós Király, The Vienna Convention on International Sales of Goods and the Bitcoin 16 (5) US-China Law Review 179 (2018); Neil Tiwari, The Commodification of Cryptocurrency 117 (3) Michigan Law Review 611 (2018).

159 Andrei Kirilenko, Bitcoin and Blockchain: How Do You Regulate Something as Intangible as a Cryptocurrency?, <https:/www.imperial.ac.uk/business-school/knowledge/finance/Bitcoin-blockchainregulate-something-intangible-cryptocurrency/> (accessed 17 October 2019). 
terminology relating to the technology. ${ }^{160}$ This problem is a recurrent one in any field where technology or practice is actively developing. ${ }^{161}$ The language evolves quickly for both substantive and non-substantive reasons, which makes it difficult for regulators to draft precise and comprehensive legislation. ${ }^{162}$ One obvious example is blockchain technology itself, which may be referred to as 'the blockchain' or 'blockchain', 'distributed ledger technology', ${ }^{163}$ 'distributed registry technology', ${ }^{164}$ 'shared ledger technology', ${ }^{165}$ 'consensus ledger technology', 166 'mutual distributed ledger technology', ${ }^{167}$ and a 'decentralised' or 'distributed database'. ${ }^{168}$ There is a similar variety of terms relating to public and private blockchains, to parties involved in operating the databases or ledgers and to digital assets.

Some of the terms used by countries to reference cryptocurrency include 'digital currency' (Argentina, Thailand, and Australia), ${ }^{169}$ 'virtual commodity' (Canada, China, and Taiwan), ${ }^{170}$ 'crypto-token' (Germany), ${ }^{171}$ 'payment token' (Switzerland, United Kingdom), ${ }^{172}$ 'cyber currency' (Italy and Lebanon), ${ }^{173}$ 'electronic currency' (Colombia and Lebanon), ${ }^{174}$ and 'virtual asset' (Honduras and Mexico). ${ }^{175}$ This disparity in terms has two consequences: first, it can make regulators dependent on industry experts and thereby increase the risk of regulatory capture and under-regulation. ${ }^{176}$ Second, if restrictions are proposed for a particular cryptocurrency, it could evade legislation simply by changing its form or name. A diverging terminology between regulatory regimes could further lead to inconsistent regulation that could be exploited by regulated parties through forum shopping and regulatory arbitrage. ${ }^{177}$

160 Angela Walch, The Path of the Blockchain Lexicon (and the Law) 36 Review of Banking \& Financial Law 728 (2017).

161 Ibid.

162 Ibid.

163 Andrea Pinna \& Wiebe Ruttenberg, Distributed Ledger Technologies in Securities Post-Trading, $<$ https://www.ecb.europa.eu/pub/pdf/scpops/ecbop172.en.pdf $>$ (accessed 18 October 2019).

164 Nikolay Mrochkovsky, Introduction of the Distributed Registry Technology into the Company's Management System 212 MATEC Web of Conferences (2018).

${ }_{165}$ Merlinda Andoni et al., Blockchain Technology in the Energy Sector: A Systematic Review of Challenges and Opportunities 100 Renewable and Sustainable Energy Reviews 144 (2019).

166 Pinna \& Ruttenberg, supra note 147.

167 Michael Mainelli \& Alistair Milne, The Impact and Potential of Blockchain on the Securities Transaction Lifecycle, <https://ssrn.com/abstract=2777404> (accessed 18 October 2019).

168 GSMA, Blockchain - Operator Opportunities (2018).

169 The Law Library of Congress, Regulation of Cryptocurrency Around the World, <https://www. loc.gov/law/help/cryptocurrency/world-survey.php> (accessed 18 October 2019).

170 Ibid.

171 Ibid.

172 Ibid.

173 Ibid.

174 Ibid.

175 Ibid.

176 Andrew Baker, Restraining Regulatory Capture? Anglo-America, Crisis Politics and Trajectories of Change in Global Financial Governance 86(3) International Affairs 647, 663 (2010).

177 Angela Walch, The Path of the Blockchain Lexicon (and the Law) 36 Review of Banking \& Financial Law 734, 735 (2017). 
It could also increase the costs for users of the market and stifle innovation. ${ }^{178}$ Standardisation of the blockchain industry is needed as well as better tools that have been certified by governments to manage crypto accounting. ${ }^{179}$ ISO standards could define a common lexicon on what is a blockchain and although this standardisation would not be mandatory, it could allow a certified company to use it as a marketing argument, and to play an active role in ensuring other actors also comply with the standards.

\subsubsection{Substantial Problems in Experimenting with the Regulation of a Dispersed Architecture}

Beyond terminology problems, blockchain regulation is made difficult by the dispersed architecture of the technology, the lack of obvious links between an event occurring on the blockchain and a particular jurisdiction, and the increasing numbers of autonomous entities based on smart contracts, which are not traditional legal entities. ${ }^{180}$ These challenges are particularly evident for public blockchains, especially when commercial transactions incorporate smart contracts and artificial intelligence. ${ }^{181}$ Conventional means could suffice to solve legal challenges related to private blockchains, as this kind of DLT is simply a decentralised register managed by a closed group of entities. ${ }^{182}$ Without any legal precedent in this area, regulators should resort to experiment and address issues on a case-by-case basis. Additionally, they should take a coherent approach and anticipate solutions to problems that are likely to emerge in the future. Such a coordinated and visionary approach is not simple because individual states may not coordinate their legislation, and actions may appear disorganised. The main global attempt to discuss the issue in depth was at the G20 summit held in Buenos Aires in 2018, where the Financial Stability Board called for 'further international coordination' and 'enhanced monitoring' ${ }^{183}$ Leaders resolved to address money-laundering and terrorist financing risks by regulating crypto-assets in line with Financial Action Task Force (FATF) standards. ${ }^{184}$ The first global standards were introduced in June 2019 by the FATF but the way these new requirements are actually implemented still needs to be assessed. ${ }^{185}$

\footnotetext{
178 See AFNOR, Coming Soon: ISO Standards for Blockchain Technology, <https:/www.afnor.org/ en/news/coming-soon-iso-standards-for-blockchain-technology/> (accessed 18 October 2019).

179 Ibid.

${ }^{180}$ Krzystof Wojfyto, How May We Regulate the Blockchain?, <https://newtech.law/en/how-maywe-regulate-the-blockchain/> (accessed 4 December 2019).

181 Donna Dillenberger, Petr Novotny, Qi Zhang, Praveen Jayachandran, Blockchain Analytics and Artificial Intelligence 99 IBM Journal of Research and Development 11 (2019).

${ }^{182}$ Krzystof Wojfyto, How May We Regulate the Blockchain?, <https://newtech.law/en/how-maywe-regulate-the-blockchain/> (accessed 4 December 2019).

${ }^{183}$ G20 Summit Argentina, G20 Leaders' Declaration: Building Consensus for Fair and Sustainable Development, <http://www.g20.utoronto.ca/2018/2018-leaders-declaration.html $>$ (accessed 4 December 2019).

184 Ibid.

185 FATF, Money Laundering Risks from "Stablecoins" and Other Emerging Assets, <https://
} 


\section{Conclusion}

One of the main features of cryptocurrency is that it has decentralised elements that can bypass government regulation. This is a benefit that enhances exchanges at the cross-border level, enabling bureaucratic barriers erected by states to be avoided. The exaggerated promotion of cryptocurrency in recent years is not necessarily linked with the perceived positive benefits, and its fall from favour is largely due to a lack of governance, because many of its uses have been associated with illegal or illegitimate activities. A review of the current regulatory approach among major jurisdictions shows that cryptocurrency, rather than providing a solution to a legal problem, generates further legal and regulatory problems that need to be tackled. A decentralised approach to regulation would not provide market confidence but could instead create further opportunities for illegitimate and illegal operators to explore ways to benefit from cross-border transactions. It is argued that public chain cryptoassets should not be recognised as a legal tender, however, money laundering law and tax law should apply to capture illegal activities by using cryptoassets. Cryptoasset exchanges should comply with KYC and AML requirements to for anti-money laundering and fraud prevention. In order to protect the consumers, the market abuse regime should also apply to exchanges. Exchanges should have a system to protect consumers by safekeeping the 'points/tokens' in the user's wallet. Furthermore, cryptocurrency being used as benchmark should be banned for retail investors due to the absence of a reliable basis for valuing the underlying assets, the extreme volatility in cryptoasset prices movements, the frequent market abuses and financial crimes in the secondary market for cryptoassets, as well as the inadequate product knowledge of most retail investors. Finally, if merchants already accept cryptoassets as payment, consumers should be warned about the associated risks before contracting. Consumer protection law should apply to contracts in which cryptoassets have been used as a means of payment. If a merchant fails to deliver the goods, the consumer could then demand compensation with fiat money.

www.fatf-gafi.org/publications/fatfgeneral/documents/statement-virtual-assets-global-stablecoins.html> (accessed 4 December 2019). 